\title{
An Electro-Dynamic Energy Harvester Supplying a Wireless Sensor Node
}

\author{
Florian Maier, Stefan Dierneder, Günther Weidenholzer, Achim Berger \\ Austrian Center of Competence $\mathrm{GmbH}$, \\ Altenbergerstrasse 69, 4040 Linz, Austria, office@accm.co.at
}

\begin{abstract}
:
Nowadays, most wireless sensor nodes are supplied by electrical energy with built-in batteries or wireconnected to an external electrical energy source. Additionally, in some industrial applications wireless sensor nodes are hermetically sealed into housings even without any electrical conductive connections through the shape. Compared to batteries an installed energy harvesting module requires no costly maintenance, but its power output depends on the used energy source, during data transmission the instantaneous power input of wireless sensor nodes surmounts the energy harvester's average power, and has higher production cost. This paper presents a wireless sensor node supplied by an energy harvesting module. A frequency adjusted and by an electro-dynamic converter only lightly damped spring/mass oscillator converts mechanical energy of bulk vibrations into electrical power. Both the converter properties and the data transmission are modeled in the paper. The discussed energy harvester module was model based designed and built in our lab, has a resonance frequency of about $50 \mathrm{~Hz}$, provides an electrical average power of more than $50 \mathrm{~mW}$ by a vibration amplitude of $0.1 \mathrm{~mm}$, and goes up to $200 \mathrm{~mW}$ at increased excitation. In the current design state the wireless sensor node transmits acquired data from both, a $3 \mathrm{D}$ acceleration and a temperature sensor. The data transmission is scheduled by a proprietary ALOHA protocol and works reliable for distances up to 10 meter (indoor) with a transmission rate of about 150 Bytes per second.
\end{abstract}

Key words: energy harvesting, wireless sensor node, frictionless linear guidance, mechanical vibrations, resonance

\section{Introduction}

In many industrial applications the variety of sensors in use is expanding. This is because performances parameter steadily will be improved, such as higher production speed, increase of product quality and energy efficiency, or enlargement of service intervals and preventive maintenance. A state of the art application, for example, is the investigation of bearing bulk vibrations that allows a prediction of out of order events and helps avoiding unexpected machine outages. The high number of applied sensors produces big amount of costly wiring. Additionally, for some applications wiring is difficult or impracticable, because of harsh environment properties. Currently, more often wireless sensor nodes will be used to manage the present challenges. Collected data is transmitted by electromagnetic waves from the wireless sensor nodes to the master node, which provides the acquired data e.g. to a bearing control resp. maintenance office. In addition, wireless data transmission avoids the risk of wire breaking $[1,2,3]$.
Both, inevitable battery replacement and additional wiring effort causes high costs. Hence, there are only built-in power supplies useable such as appropriate energy harvesting methods, or high capacity batteries [1].

The data transmission via wireless communication consumes certain amount of energy. The internal power supply is a rather involved problem, which is still insufficiently solved. Popular power sources are batteries, but those face limited working time, depending on its capacity, an unsolved deposing problem after depleting, and resource shortness on matter used in battery manufacturing. Planning of maintenance becomes more and more important for reducing unwanted production breaks. For the external supply with electrical energy a wire-connection from net to the sensor node is used $[3,4]$.

Energy Harvesting solves some of these problems, but its applicability also depends on environmental requirements - thinking about a reliable energy source, that is usable for energy harvesters. In our case these constraints are 
the properties of mechanical vibrations such as frequency and amplitude. In the subsequent section an itemization of other useable energy sources for industrial applications is given $[5,6$, 7].

Wireless data transmission technique only makes real sense together with wireless power supplies, because the following problems are solved. At first, a compact design of the energy harvester together with wireless sensor node makes it possible to put all in one and therewith enables hermetically sealed sensor boxes. Secondly, there is limited battery energy versus the need for high energy for data transmission. Batteries show limited energy capacity, which is mainly restricted by the volume of the sensor box. The battery capacity defines the maintenance period of the wireless device, and additionally, batteries have self-discharging. Thirdly, wireless devices are independed from wiring limitations such as physical problems and installation cost [1, 3].

A subsequent power management rectifies $A C$ to DC current, stores energy surplus, and provides electrical energy in appropriate voltage level. In order to manage such power pulses, first of all, the storage capacity must fit to the temporarily increased energy demand, which is defined by the integration of average power surmount over transmitting period, and secondly, the wireless sensor nodes has to use optimized transmitting protocols that cope with lower power supply variance [4].

After this introduction and view on possible industrial applications, such as temperature and acceleration monitoring and a review of related literature, section 2 discusses some important theory about mechanical oscillators, power absorption ability, and influence of gravity. Interesting details of the wireless sensor node design are discussed in the $3^{\text {rd }}$ section. The fourth section presents the linear bearing with low frictional losses, which is a core element of the presented energy harvester. Section 5 deals with the optimization of the magnetic circuit, and section 6 discusses the implied wireless transmission of sensor data. Finally, the sections "Results" and "Conclusion" discuss the performance of the designed wireless sensor node and show some future aims.

\section{Theory Energy Harvesting using Vibrations}

The energy conversion from mechanical vibrations to electrical energy is widely discussed in $[6,7,8]$. Such energy harvesters are based on a mass/spring system with an electro dynamic converter, which is damping the system.

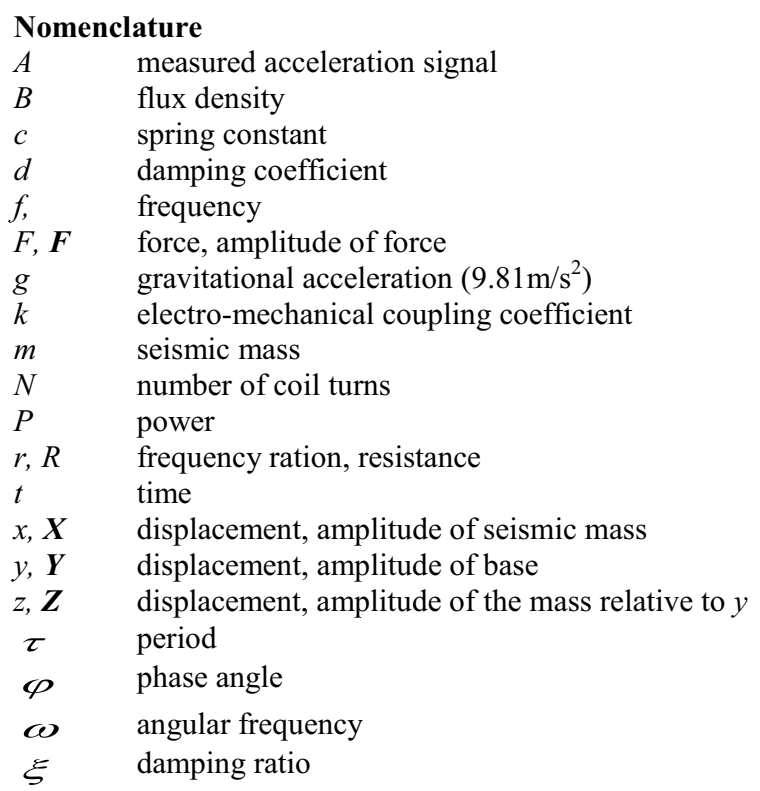

A one degree of freedom (1 DOF) lightly damped horizontal moving harmonic oscillator according to Fig. 1 will be analyzed. The absolute positions are given by $y$ (massless foundation plate) and $x$ (position of the mass $m$ ).

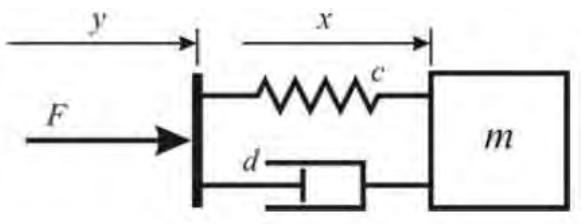

Fig. 1: Model of the excited and lightly damped oscillator.

An in advance undetermined force $F(t)$ excites the foundation plate in the way that it moves in steady state with displacement $y=Y \sin (\omega t)$. Further, the plate is connected to the mass $m$ with a hookean spring (constant $c$ ) and a damping element (coefficient $d$ ). The damping coefficient is defined by $d=\xi d_{c}$, with the critical damping $d_{c}$ and the damping ratio $\xi=2 m \omega_{\mathrm{n}}$.

A consideration of gravity is necessary for oscillators with vertical moving directions; because static deflection offsets reduce the frame preset displacement range. The gravitational force $m g$ and the spring force $c \Delta$ must be equal, $c \Delta=m g$. Consequential, the deflection offset $\Delta=m g / c=g \omega_{\mathrm{n}}^{-2}$ is proportional to gravity and reciprocal to squared natural angular frequency. For an oscillator with a displacement range of $1.5 \mathrm{~mm}$ and a supposed resonance frequency of $f_{\mathrm{n}} \geq 30 \mathrm{~Hz}$ the induced offset $\Delta$ is $<277 \mu \mathrm{m}$. The influence to the electrical output power is discussed later on.

The following equation of motion describes the dynamic of the oscillator according Fig. 1. 


$$
m \ddot{x}+d(\dot{x}-\dot{y})+c(x-y)=F(t)
$$

A state transition from system states $x$ and $y$ to state $z$ is made by the substitution $x-y=z$, and its derivatives with respect to time.

$$
m \ddot{z}+d \dot{z}+c z=m \omega^{2} Y \sin (\omega t)
$$

Finally, the (in time state $z$ ) created equation of motion is quite compact. Rearrangement determined the force $F(t)=F \sin (\omega t)$ (with $\boldsymbol{F}=m \omega^{2} Y$ and $\left.\omega=2 \pi f\right)$ that is proportional to the mass $m$ and the acceleration of the foundation plate. The solution of eq. (2) is the harmonic time function

$$
z=Z \sin (\omega t-\varphi)
$$

In steady state condition the motion state $z$ oscillates with angular frequency $\omega$ and phase angle $\varphi$. The amplitude $Z$,

$$
Z=\frac{m \omega^{2} Y}{\sqrt{\left(c-\omega^{2} m\right)^{2}+d^{2} \omega^{2}}}
$$

depends to a great extent on the angular frequency of the external force $F(t)=\boldsymbol{F} \sin (\omega t)$. Other amplitude dependencies are the mass $m$, the spring constant $c$, the damping coefficient $d$, and, the foundation plate amplitude $Y$. The amplitude of the non-linear function $Z$ has a peak at its natural angular frequency $\omega=\omega_{\mathrm{n}}$.

Equation (5) gives the instantaneous power $P_{\text {inst }}$ dissipated by the damping element $d$.

$$
P_{i n s t}=d \dot{z}^{2}=d \omega^{2} Z^{2} \cos ^{2}(\omega t-\varphi)
$$

The power $P_{\text {inst }}$ is the product of the damping coefficient $d$ and the velocity value, which is derived from eq. (3).

The average power $P_{a v}$ is calculated by time mean of the instantaneous power eq. (5) and is given by eq. (6).

$$
P_{a v}=\frac{d \omega^{2} Z^{2}}{2}=\frac{d \omega^{2} Z \cdot m \omega^{2} Y}{2 \sqrt{\left(c-m \omega^{2}\right)^{2}+d^{2} \omega^{2}}}
$$

The aim is a useful relation for the power flow $P_{\text {flow }}$, which defines the mechanical power that is transformable at oscillator resonance from the environment into the energy harvester system [6, 7]. Based on eq. (6) two variable replacements are necessary. Firstly, the parameter $Z$ is replaced by $Z_{\max }$, which considers the maximum amplitude $Z$ of mass $m$ movement. Secondly, the angular frequency $\omega$ is replaced by the natural angular frequency $\omega_{\mathrm{n}}$ at resonance state.

$$
P_{\text {flow }}=\frac{m \cdot \omega_{n}^{3} \cdot Y \cdot Z_{\max }}{2}
$$

The power $P_{\text {flow }}$ is directly proportional to the mass $m$, to the cube of the angular frequency $\omega_{\mathrm{n}}$, the maximum possible moving amplitude $Z_{\max }$ of the mass $m$, and the excitation amplitude $Y$, but is independent from the damping coefficient $d$, see [6, 7, 8].

The normalized power value $\hat{P}_{a v}$ shows the amount of power flow $P_{\text {flow }}$ from the surrounding vibrating environment into the energy harvester system around the resonance state.

With the further substitution of the damping coefficient $d=\xi d_{c}$, the equation of the normalized average power eq. (8) reads as following:

$$
\begin{gathered}
\hat{P}_{a v}=P_{a v} / P_{\text {flow }} \\
\hat{P}_{a v}=\frac{2 P_{a v}}{m \omega^{3} Y Z_{\max }} \approx \frac{2 \xi\left(\omega / \omega_{n}\right)^{4}}{\sqrt{\left(1-\left(\omega / \omega_{n}\right)^{2}\right)^{2}+4 \xi^{2}\left(\omega / \omega_{n}\right)^{2}}}
\end{gathered}
$$

The right term of eq. (8) is shown in Fig. 2, where one can see that in the resonance state the normalized average power is independent of the damping ratio $\xi$.

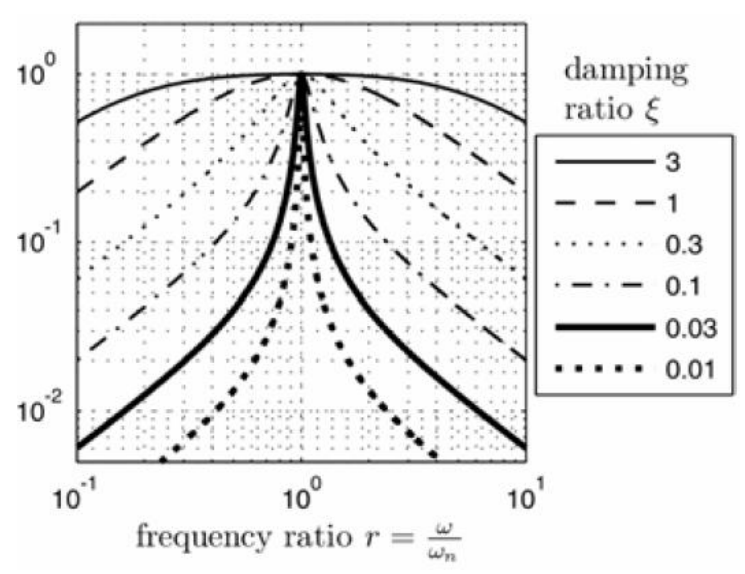

Fig. 2. Variation of the normalized average power $\hat{P}_{a v}$

\section{General Concept of the Energy Harvester}

The design of the wireless and battery less sensor node, see [8], is rotation-symmetric. 
Figure 3 shows a cross section through the 3DCAD model. The housing of the sensor node consists of a foundation plate and a covering cap, sealed together. This prevents unwanted matter penetration, dust particle or fluids, but allows penetration of electromagnetic radiation needed for wireless data transmission. The bottom of the foundation is mechanically stiff connected to the vibration source.

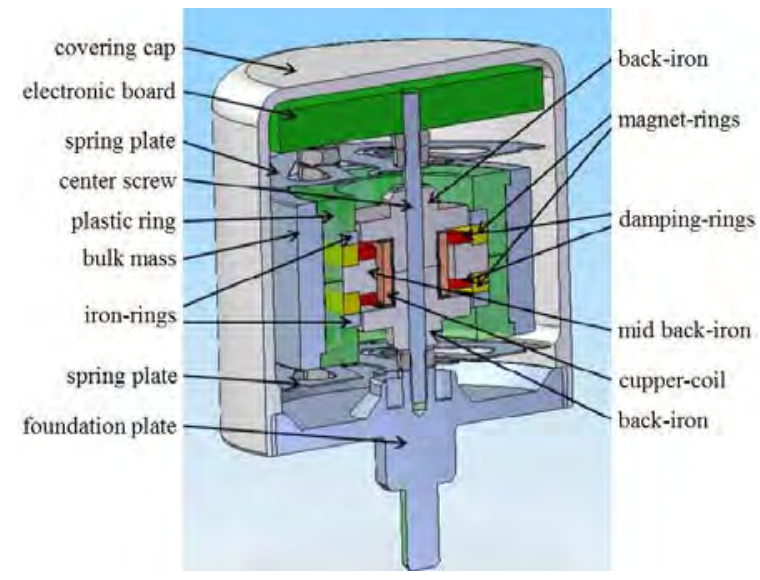

Fig. 3. 3D CAD model of the sensor node

Within the housing on top of the foundation plate a headless center screw locates lower spring plate (Fig. 4), lower back iron, cuppercoil, upper back iron (example of flux density see Fig. 5), upper spring plate (Fig. 4), and the electronic board. Additionally, some nuts, and distance elements carry out the vertical site of the main components.

\section{Linear Bearing with Low Frictional Losses}

Figure 4 shows the worked out spring plate in one deflected position, [8]. A stack of these spring plates fulfills two for the energy harvester necessary functions. Firstly, a linear bearing with low frictional losses and secondly a spring, which provides the oscillator's restoring force. The spring constant can be tuned in discrete steps by stacked spring elements. Additionally, a thickness change of the metal sheet allows fine adjustment of spring constant.

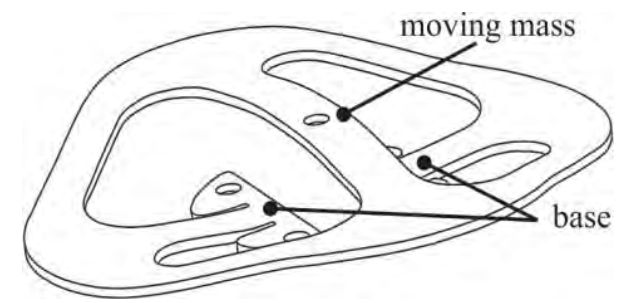

Fig. 4 View on the special spring plate, which is patented and which can be manufactured cheap and easily by laser-cutting technique.

\section{Optimization of Magnetic Circuits}

The rotation-symmetric design of the magnetic circuit consists of the back iron, two permanent magnet rings, the air gaps and the copper coil, see Fig. 3 and Fig. 5. Additionally, Fig. 5 shows the qualitative flux density in the parts of the designed energy converter.

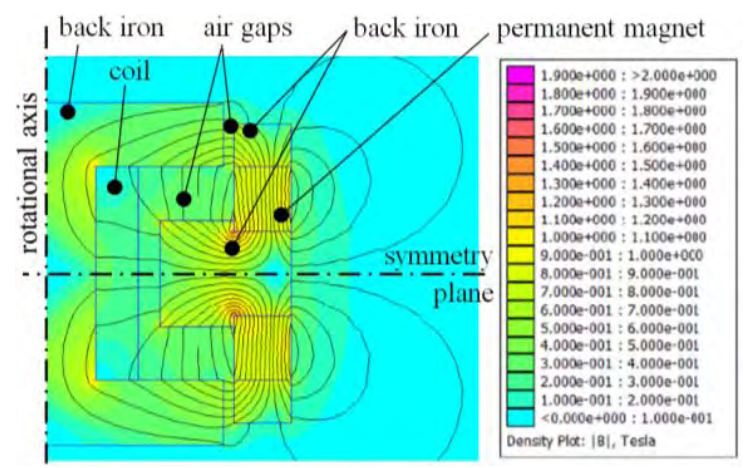

Fig. 5. Color coded simulated field patterns of the current-less electro dynamic converter. The pattern of the probed central position state is symmetrically to the horizontal plane.

Figure 7 presents three more flux patterns, which look quite unequal. This is because the bulk mass with permanent magnet-rings is positioned out of the center. A terminal voltage is induced due to the change of the flux linked to the coil. In Fig. 6 the results of negative restoring forces are shown for four states $z=\{0$, $0.5,1,1.5\} \mathrm{mm}$ (blue circles). The approximated force function shows the flux-gained force, which would de-tune the oscillator's natural frequency (destabilizing stiffness is about 14.7 $\mathrm{N} / \mathrm{mm}$ ). Diagram in Fig. 6 shows the oscillator's restoring force as the summation of magnetic force and the mechanical spring stiffness.

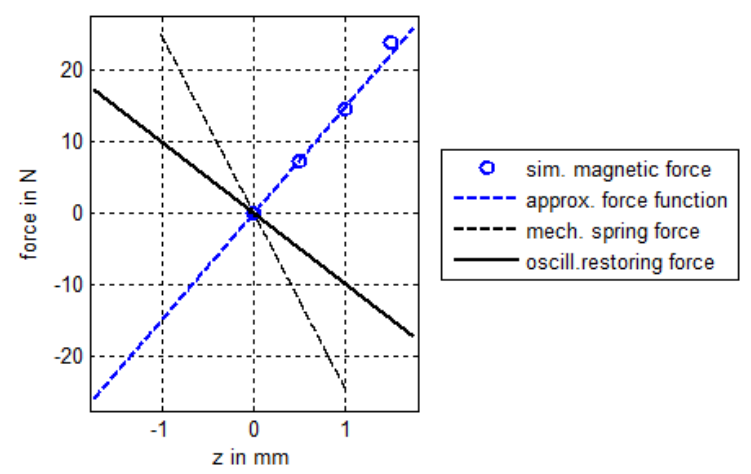

Fig. 6: (blue circles) Calculated forces based on the flux patterns (Fig. 5 and Fig. 7); (blue dashed line) linear approx. force function; (black dashed line) spring force (Fig. 4); and (black line) function of the oscillator's restoring force. 
Fig. 7: Flux density results; The state $z(t)$ indicates a deflection between interior back iron and the outer bulk mass (mid back-iron, perm. magnet rings and iron rings). The forces caused by flux are shown in Fig.6. These forces work against the spring plates.

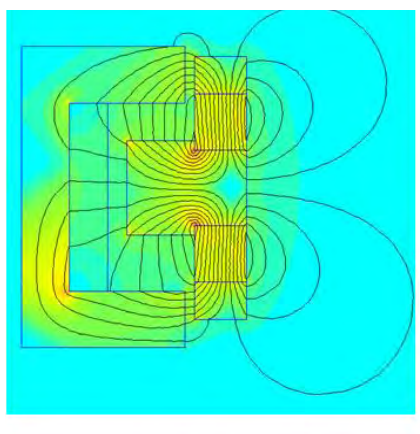

(a), $z=0.5 \mathrm{~mm}$

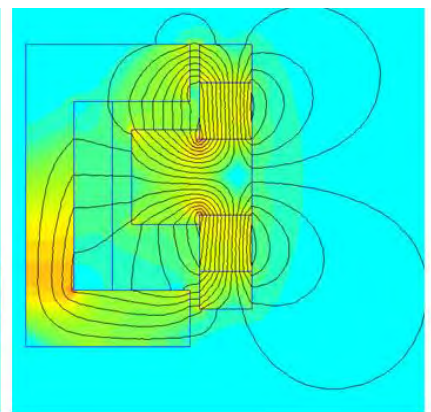

(b), $z=1.0 \mathrm{~mm}$

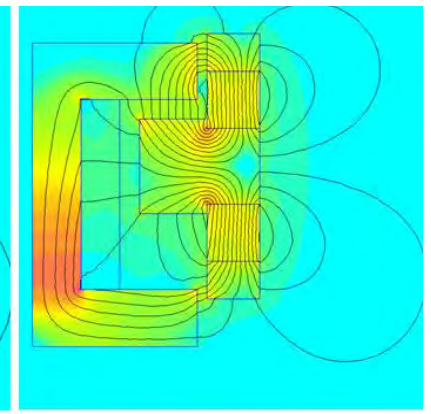

(c), $z=1.5 \mathrm{~mm}$

The size of the magnetic circuit is tuned within limits set by the mechanical structure. The aim of the iterative design process was the maximization of power output, besides minimizing the transformer volume, a reduction of expansive permanent magnet material and copper wire. Despite quite big air gap high gradients in coil flux are possible. The quality of the linear guidance specifies the size of the air gap.

\section{Wireless Transmission of Sensor Data}

For the wireless communication only a limited amount of electrical energy is available. Therefore, optimized transmission protocols for energy aware communication have been developed and are in use, see [4]. In the first design step a robust concept has been applied. Later on some improvements have been discussed for further reduction of the energy consumption. The sensor node in the described WSN transmits acquired data from a 3D acceleration sensor or an on board temperature sensor. In the proposed protocol, the authors focus on the transmission of acceleration data, sampled with $500 \mathrm{~Hz}$ at 8 bit resolution and buffered in blocks of 150 samples. The base station requests about one data block per second.

Figure 8 shows the implied communication between sensor (client) and master node. At the beginning (top) the client asks for commands from the master node, but it doesn't answer during the request period. The master replies the second request attempt with the instruction "CMD: ACC X". The client starts data acquisition from the acceleration sensor, divides collected data in three packets, transmits the first data packet to the master node, and subsequent waits for an acknowledgment, in short "ACK". In case of a successful data transmission the client sends the second data packet and so on. If the "ACK" will not be received in a specified time interval, the client sends the data packet again. The

delay time between data packet transmission depends on the actual power situation of the energy harvester. In case of energy shortage the transmission starts time-delayed, until enough electrical energy is stored in the buffering system so that the wireless sensor node can work reliably again.

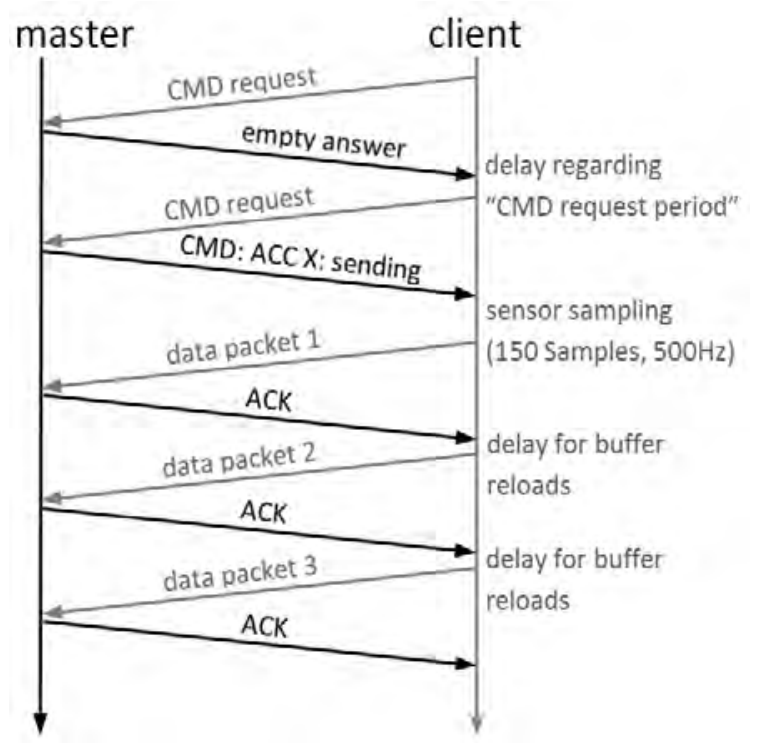

Fig. 8. The diagram demonstrates the communications during measuring interval.

\section{Results}

The sensor node acquires data (sample rate $500 \mathrm{~Hz}$ ) of 3D accelerations and transmits them to the master node with a sensor transmission rate of about 150 Bytes per second. As mentioned in the paper, enough available electrical power (offered by the integrated energy harvester) is essential for the reliable work of the wireless sensor node. The complexity and non-linear behavior of the implemented electric circuit (consisting of the components: electrical voltage rectifier, voltage stabilizer, power management, energy storage elements, and SRD (short range device) transceiver) necessitates an experimental analysis of the power consumption. 
The experiment was dedicated to two interesting questions. On the one hand side the wake-up power, the integrated energy harvester supplies to the sensor node electronics. In this working state the power management already starts working, but the sensor node with data acquisition and transmission is still inactive. On the other hand, the so called critical power, which indicates the required minimum power, above the sensors work and the sensor node acquires data and transmits them reliably. The experimental arrangement consists of the electronic board of the wireless sensor node and the energy harvester shown in Fig. 3. The electrical circuit is supplied by the energy harvester via two wires conducting the induced voltage with sinusoidal characteristics as shown in Fig. 9. The supplied power is measured by acquiring both voltage and current with a transient recorder. In order to find out the wakeup power and critical power the external moving frequency was set to $52 \mathrm{~Hz}$. During the experiment the sinusoidal vibration amplitude was increased by a rate of less than $10 \mu \mathrm{m} /$ $\min$.

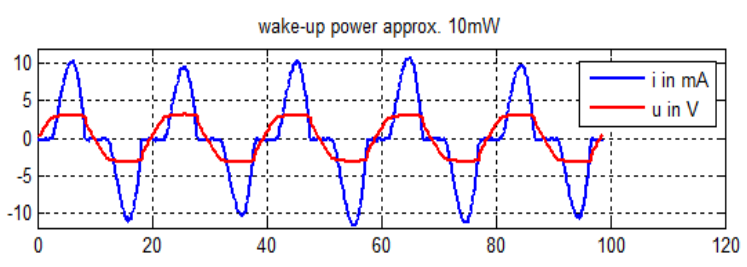
critical power approx. $25 \mathrm{~mW}$

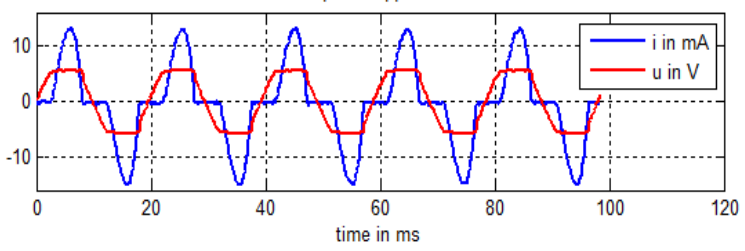

Fig. 9. Induced voltage and current signals of the energy harvester

The results of the experiment were that the wake-up power is approximately $10 \mathrm{~mW}$, which has been calculated from the acquired signals, voltage and current, shown in the upper diagram of Fig. 9. Further increase of the mechanical excitation raises the power of energy harvester transferred to the sensor node electronics. The second part of Fig. 9 shows the acquired signals, which result in approx. $25 \mathrm{~mW}$ critical power. Above this power value the sensor node samples and works reliably.

\section{Conclusion}

First, the paper presents the motivation of hermetically sealed wireless sensor nodes without any external electrical contacts. With wireless sensor nodes many physical parameters e.g. in industrial plants can be observed, in spite of harsh environmental properties. The integrated energy harvester module allows much longer maintenance intervals compared to battery supply. Besides, the paper gives necessary theory about oscillator tuning and a special view on the design. The wireless sensor node is a real mechatronical example consisting of mechanical design, optimization of the electro dynamic transformer's magnetic circuit, and wireless communication not only in theory, also implemented and tested in our labs. The built sensor node (including the energy harvester) samples sensor values and transmits the data reliably beyond an electrical power of approx. $25 \mathrm{~mW}$. Such energy can be typically harvested on electric motors above $2 \mathrm{~kW}$ electrical power or on bearing frames with comparable mechanical frame vibrations.

\section{Acknowledgement}

The authors acknowledge the sponsoring of this work by the Austrian Center of Competence in Mechatronics $\mathrm{GmbH}$.

\section{References}

[1] S. Priya, D.J. Inman, Energy Harvesting Technologies, book ISBN 978-0-387-76463-4 Springer Science+Business Media LLC, 233 Spring Street, New York, NY 10013, USA

[2] PMG Perpetuum: (“http://www.perpetuum.co.uk")

[3] S. Mahlknecht, S.A. Madani, On Architecture of Low Power Wireless Sensor Networks for Container Tracking and Monitoring Applications, Proceedings of 2007 IEEE International Conference on Industrial Informatics, vol. 1, INDIN07, 2007, pp. 353 - 358.

[4] A. Berger, A. Pötsch, A. Springer, TDMA Proposals for Wireless Sensor Networks for Highly Reliable and Energy Efficient Data Collection in an Industrial Application

[5] F. Ziegler, Mechanics of Solids and Fluids, book ISBN 0-387-94399-4, New York/Vienna, Springer-Verlag, (1991).

[6] C.B. Williams, R.B. Yates, Analysis of a microelectric generator for microsystems, Elsevier Sensors \& Actuators A Physical, vol. A52, no. 13, 1996.

[7] N.G. Stephen, On energy harvesting from ambient vibration, Elsevier Journal of Sound and Vibration, vol. 293, pp. 409-425, 2006

[8] F. Maier, M. Sturmlechner, S. Dierneder, Novel Energy Harvesting with Low Friction Losses, 6th International Multi-Conference on Systems, Signals and Devices, Djerba, Tunesien 2009, 32009, ISBN: 978-1-4244-4346-8 (CD) 